\section{Mental disorder and perceived threat to the public: people who do not return to community living}

\author{
LIZ JAMIESON and PAMELA J. TAYLOR
}

Previous studies of patients with mental disorder after discharge from high-security hospitals have focused on reconvictions and readmissions, implying that, once discharged, they are 'at risk' in the community. Buchanan (1998) noted in his follow-up study of patients discharged in 1982-1983 that 'some patients will have spent all or part of the follow-up period in hospital or prison', but did not specify further. Norris (1984) identified 423 men who left Broadmoor between 1974 and $1981,22 \%$ of whom over 7 years did not leave lesser security; it remains unclear whether this continued hospitalisation was protective against reoffending. Subsequently, the Mental Health Act 1983 (MHA) granted more opportunities for challenging hospital detention. Following an interdepartmental government review of services (Home Office \& Department of Health and Social Security, 1975) new medium secure hospital places were created, many by the mid-1980s (Snowden, 1985). This was in the context of otherwise increasing emphasis on community treatment for psychiatric patients.

Our study was designed to conduct the longest possible follow-up of place of residence for all patients discharged from special hospital in $\mathbf{1}$ year within the current legislative and service framework. The principal hypothesis was that no patient leaving special hospital would remain indefinitely institutionalised. A subsidiary hypothesis was that reoffending would be associated with community living. Were a continuously institutionalised group to be identified, it was proposed to test demographic, disorder and offending characteristics at the time of admission and departure as possible early indicators of special rehabilitative need.

\section{METHOD}

\section{Sample}

All patients who were discharged in 1984 from Broadmoor, Ashworth (then Park
Lane and Moss Side) and Rampton special hospitals were included in the study.

\section{Data extraction}

The special hospitals' case register was used to identify patients in this discharge cohort. The register was set up in 1972 and holds admission and discharge details, as well as socio-demographic and criminological data, on all special hospital patients admitted since then (and more limited data on the 1977 resident cohort). Data are collected for the case register from both hospital and other records and from patient interview.

Mental Health Act 1983 classification of mental disorder is the principal indicator of diagnosis. These classes do map closely onto diagnostic categories as defined in ICD-10: mental illness on psychotic disorders, mainly schizophrenia; psychopathic disorder on personality disorders; (severe) mental impairment on (severe) mental retardation (Taylor et al, 1998).

In regard to the index offence, 'none' implies that there was no index charge or conviction, not that there had been no violent or dangerous behaviour. 'Property' offences relate to arson or serious property damage, and 'other' offences include burglary or attempt, theft, non-indictable assault, breach of the peace and other indictable offences.

\section{Length of follow-up}

Data were recorded from the date of formal discharge from special hospital (or date of departure if leaving on trial leave) until the last known placement, or to the census date of 31 December 1995 or until death, whichever was earlier (maximum 12 years).

\section{Sources of follow-up data}

(a) Data relating to deaths, in-patient periods of over 2 years, and local family health service authorities with which patients were registered were obtained from the Office for National Statistics.

(b) Details of readmissions to special hospital were recorded from the special hospitals' case register.

(c) For patients under Home Office Restriction Orders (sections 37/41, MHA), placement data were collected from the Home Office's Mental Health Unit files. 
(d) For patients who were not on restriction orders, and for others as soon as they achieved discharge from such orders, information was obtained by writing to the place to which the patient had been discharged and asking whether the person was still resident and/or in the care of someone at that place. If so, the name of that person was requested. If not, the name of the person to whom care had been transferred, the date of transfer and/or the place of transfer were requested. A letter was then written to such placement, and so on until, as far as possible, all placements for each person for the period had been identified. If no reply was received to a letter, a follow-up call was made to the organisation's records department in each case.

Where exact dates for placements (hospital, prison or community) were not known, the last day of the month, where known, was used. Otherwise, the last day of the year was used. The date of leaving prison was assumed to be the earliest date of release, unless known otherwise. If patients left hospital and entered the community on trial leave rather than formal discharge, they were treated as being in the community unless a new episode of institutional admission was recorded. For any individual not traced by this method, an additional search of the computerised electoral roll for the year 2000 was made to see if the patient was living in the community. (Earlier computerised electoral roll listings were not obtainable.)

(e) Reconviction was taken as a measure of reoffending. Data were obtained from the Offenders' Index, which is part of the Research and Statistics Division of the Home Office. It holds a record of all standard list convictions from 1963. In cases where there was no trace of reconviction from the Offenders' Index, but the Mental Health Unit's (Home Office) records detailed a reconviction, the latter information was included.

\section{Analyses}

(a) Multinomial logistic regression is useful for situations in which it is necessary to classify subjects based on values of a set of predictor variables. A multinomial logistic regression analysis was carried out using STATA v.6 for PC (StataCorp, 1997) to examine the variables on discharge associated with membership of each of the four groups. These groups consisted of: those who died without reaching the community; those still institutionalised after 12 years; those who died after some time in the community; and those who were alive in the community. The analysis used as a base category the group of those alive in the community, to which the other groups are compared. The independent variables were age on discharge, length of stay and MHA classification of disorder on discharge.

(b) Logistic regression was used to examine the independent effects on the likelihood of attaining community status at all of a number of variables, including length of follow-up, MHA classification of disorder on discharge, gender and length of previous stay in special hospital. Age on discharge was not included as this is significantly correlated with length of stay.

(c) Logistic regression was used to examine the independent effects on the likelihood of attaining community status at all of a number of variables, including MHA classification of disorder, age on admission and gender.

\section{RESULTS}

\section{General characteristics of the cohort}

There were 223 patients in the discharge cohort of 1984,183 men (82\%) and 40 women $(18 \%)$. In this cohort 17 were technical discharges $(7.6 \%)$, because they died in special hospital that year.

Of the 206 patients available to followup, 198 had either a full follow-up record for the 12 years $(n=93 ; 47 \%)$ or, if follow-up was not complete, it was at least possible to determine whether community living had been achieved or not $(n=105$; $53 \%)$. One patient was repatriated to his country of origin and no further information could be traced. In 7 other cases (3\%), 2 of whom died during follow-up, it was impossible to say whether a community placement was attained or not; the remaining 5 patients did not appear on the national death register, nor on any central National Health Service record, nor in official criminal statistics. It was assumed that they were alive and living in the community. Nevertheless, because of the possibility of other explanations, including human error on the national databases or use of aliases by these patients, these 7 were treated as 'not knowns'. Thus, together with the repatriated patient, 8 patients were excluded from further analysis and there was a total cohort of 215 for study (inclusive of the deaths in special hospital).

This discharge cohort was larger than the average for 1986-1990 ( $n=183)$ but similar to the average for the next 5 years (1991-1995) ( $n=224)$. Between 1986 and 1995, however, there was an increase in the discharge of patients admitted on remand orders (pre-trial or pre-sentencing) from $14 \%$ to $16 \%$ respectively of the total discharged in each year, compared with only 2 patients from the 1984 discharge cohort. This meant that in 1984 the discharge cohort size of patients who had remained in special hospital for treatment was larger than the average for the subsequent decade. The distribution by gender, nature of index offence, previous offending, death in special hospital and length of stay up to the point of discharge was, however, typical of cohorts between 1986 and 1995 (Butwell et al, 2000; Jamieson et al, 2000).

Of the 198 patients with some followup, $179(90 \%)$ were discharged to the community at some point (the community group). However, $19(10 \%)$ continued to live in other hospitals or prisons, or returned to special hospital without any residential community time up to either the date of their death or to the census date of 31 December 1995 (the institutional group).

\section{Deaths in special hospital}

Of those who died in special hospital during 1984, 10 were men $(6 \%$ of men in the discharge cohort) and 7 were women ( $18 \%$ of women in the discharge cohort). This gender difference was significant $\left(\chi^{2}=6.8\right.$, d.f. $\left.=1, P=0.009\right)$.

\section{Deaths outside special hospital}

There were a further 32 deaths among the other departures (whose community career was known) over the 12 years of the study, with no significant male/female differences overall, within or outside institutions. Of these deaths, 7 occurred before the patients had reached community living; there had been no immediate prospect of discharge to the community in any of these cases. There was only 1 
Table I Discharge characteristics and discharge outcome of the 1984 discharge cohort' ${ }^{\prime}$ of high-security hospital patients ( $\left.n=215\right)$

\begin{tabular}{|c|c|c|c|c|c|}
\hline \multirow[t]{4}{*}{ Patient characteristics } & \multicolumn{4}{|c|}{ Discharge outcome group } & \multirow[t]{4}{*}{$P$} \\
\hline & \multicolumn{2}{|c|}{ Institutional group ${ }^{2}$} & \multicolumn{2}{|c|}{ Community group ${ }^{3}$} & \\
\hline & Dead & Alive & Dead & Alive & \\
\hline & $n=24$ & $n=12$ & $n=25$ & $n=154$ & \\
\hline \multicolumn{6}{|l|}{ Gender, $n$ (\%) } \\
\hline Male & $16(67)$ & $12(100)$ & $21(84)$ & $127(83)$ & \\
\hline Female & $8(33)$ & 0 & $4(16)$ & $27(17)$ & NS \\
\hline \multicolumn{6}{|l|}{ Disorder, $n$ (\%) } \\
\hline Mental illness & $15(63)$ & $8(67)$ & $17(68)$ & $70(46)$ & \\
\hline Personality disorder & $3(12)$ & $3(25)$ & $6(24)$ & $60(39)$ & \\
\hline Severe mental impairment & $6(25)$ & I (8) & $2(8)$ & $24(15)$ & 0.07 \\
\hline \multicolumn{6}{|l|}{ Age on discharge (years) } \\
\hline Median (range) & $49.2(22.8-76.4)$ & $40.5(20.4-55.8)$ & $42.7(24-60.6)$ & 32.8 (I7.8-71.9) & $<0.001$ \\
\hline Mean & 49.0 & 39.8 & 43.6 & 34.6 & $<0.001$ \\
\hline \multicolumn{6}{|l|}{ Special hospital, n (\%) } \\
\hline Broadmoor & $6(25)$ & $2(17)$ & $6(24)$ & $40(26)$ & \\
\hline Rampton & $10(42)$ & $7(58)$ & $9(36)$ & $48(31)$ & \\
\hline Ashworth & $8(33)$ & $3(25)$ & $10(40)$ & $66(43)$ & NS \\
\hline \multicolumn{6}{|c|}{ Length of special hospital stay (years) } \\
\hline Median (range) & $17.0(|.4-4| .4)$ & $9.2(0.02-2 I)$ & $7.3(1.67-50.5)$ & $5.8(0.09-42.7)$ & 0.05 \\
\hline Mean & 17.7 & 8.8 & 10.0 & 7.5 & $<0.001$ \\
\hline
\end{tabular}

I. Of those leaving special hospital in 1984, 8 are not included in this table because no follow-up information could be traced.

2. Those with continuous residency in an institution until death or 12-year census.

3. Those who lived at some stage outside an institution.

woman in the group who did not reach the community; she died in institutional care. Of the 25 deaths in the group reaching the community, 1 occurred in hospital, after a readmission; 24 people were living in the wider community at the time of death.

Of the 24 deaths in the institutional group, 4 were unnatural deaths (two accidental and two open verdicts). Of the 25 deaths in the community group, 11 were unnatural deaths (3 suicide, 4 accidental and 4 open verdicts). This higher proportion of unnatural deaths in the community group was statistically significant $\left(\chi^{2}=4.31\right.$, d.f. $\left.=1, P=0.038\right)$, as shown in Table 1 .

\section{Indicators at discharge \\ of long-term residential needs outside high security}

Table 1 summarises this position, treating the readmission death as a death in the community group. Overall, $36(17 \%)$ of the 215 in the traced discharge cohort did not again live in the community by the end of the study; the deaths made certain that 24 of them never would.

There was a significant difference in age distribution at time of discharge, by death and community status. This was accounted for by the tendency for the institutional group to be older than the community group, and within each group the deaths to have affected those who were older at the time of discharge. Few patients, however, attained 70 years of age. The median age of death for the institutional group was 49.9 years (22.8-76.4 years) and for the community group 51.1 years (25.8-69.7 years). The survivors had a median age of 52.1 years (31.9-67.3 years) and 44.4 years (29.3-83.6 years), respectively, at the end of the follow-up. The groups varied in length of stay in special hospital, with the institutional group having stayed longer (particularly accounted for by those who died there).
The distribution appeared to differ according to class of disorder, although this did not reach statistical significance. Relatively more of the people in the group with mental illness failed to reach or survive in the community compared with the groups with developmental disorder (psychopathic disorder and (severe) mental impairment). Gender had no bearing on these equations, nor did the special hospital of principal residence.

The multinomial logistic regression analysis showed that age on discharge was significantly associated with the 'dead-ininstitution' group compared with the base category (relative risk ratio (RRR) 1.07, $95 \%$ CI $1.02-1.13, P=0.011)$ and with the 'dead-in-the-community' group (RRR $1.07,95 \%$ CI $1.02-1.12, P=0.006)$. There was no association between age on discharge and the 'alive-in-institution' group compared with the base category. Length of stay was only associated with the 'dead-in-institution' group compared with the base category (RRR 1.07, 95\% CI 
1.00-1.15, $P=0.037)$. MHA classification of disorder on discharge was not associated with any of the three groups compared with the base category.

Logistic regression to examine the independent effects on the likelihood of attaining community status at all showed that effects of length of follow-up approached but did not reach significance
$(B=-0.1362, \quad$ s.e. $=0.0809, \quad P=0.0924$, $\exp (B)=0.8727)$. Length of high-security hospital residence had a significant effect on attainment of community status $(B=-0.0572, \quad$ s.e. $=0.0270, \quad P=0.0341$, $\exp (B)=0.9444)$, with shorter length of previous special hospital stay increasing the likelihood of attaining community status.

\section{Indicators at admission of long-term residential needs outside high security}

Disorder class on admission was associated with post-discharge residential group; people with mental illness were least likely to return to community living $\left(\chi^{2}=25.2\right.$, d.f. $=9, \quad P=0.003)$. Change in MHA classification of disorder during special

Table 2 Admission characteristics and discharge outcome of the 1984 discharge cohort' of high-security hospital patients ( $n=215)$

\begin{tabular}{|c|c|c|c|c|c|}
\hline \multirow[t]{3}{*}{ Patient characteristics } & \multicolumn{4}{|c|}{ Discharge outcome group } & \multirow[t]{3}{*}{$P$} \\
\hline & \multicolumn{2}{|c|}{ Institutional group ${ }^{2}$} & \multicolumn{2}{|c|}{ Community group ${ }^{3}$} & \\
\hline & Dead & Alive & Dead & Alive & \\
\hline Total & 24 & 12 & 25 & 154 & \\
\hline \multicolumn{6}{|l|}{ Age on admission (years) } \\
\hline Median (range) & $28.6(16.8-69.3)$ & $29.3(17.9-54.1)$ & $34.7(9.6-57.4)$ & $26.2(14.8-68.9)$ & 0.006 \\
\hline Mean/s.d. & $31.3 / 11.5$ & $31.0 / 10.4$ & $33.6 / 11.5$ & $27.1 / 8.7$ & 0.004 \\
\hline \multicolumn{6}{|c|}{ Age (years) on admission by MHA classification } \\
\hline \multicolumn{6}{|l|}{ Mental illness } \\
\hline Mean/median & $33.8 / 29.9$ & $33.9 / 29.9$ & $34.5 / 33.7$ & $30.5 / 28.5$ & \\
\hline Range & $18.1-69.3 ; n=15$ & $20.4-54.1 ; n=8$ & $19.9-57.4 ; n=18$ & $16.7-69 ; n=73$ & \\
\hline \multicolumn{6}{|l|}{ Personality disorder } \\
\hline Mean/median & $28.2 / 27.9$ & $25.3 / 25.7$ & $35.0 / 41.5$ & $23.4 / 22.1$ & \\
\hline Range & $17.8-39 ; n=3$ & $|7.9-3| .8 ; n=4$ & $17.9-47.9 ; n=5$ & $14.8-37.6 ; n=57$ & \\
\hline \multicolumn{6}{|l|}{ Mental impairment } \\
\hline Mean/median & & & & $24.6 / 19.8$ & \\
\hline Range & & & & $16.4-50.5 ; n=15$ & \\
\hline \multicolumn{6}{|l|}{ Severe mental impairment } \\
\hline Mean/median & $26.6 / 26.3$ & & $22.1 / 22.1$ & $28.0 / 24.3$ & \\
\hline Range & $16.8-37.5 ; n=6$ & & $9.5-34.7 ; n=2$ & $18.3-43.7 ; n=9$ & \\
\hline \multicolumn{6}{|c|}{ Nature of index offence, $n(\%)$} \\
\hline None & $9(38)$ & $2(17)$ & $3(12)$ & $25(16)$ & \\
\hline Homicide & $4(17)$ & $3(25)$ & $5(20)$ & $29(19)$ & \\
\hline Non-fatal violence & $6(25)$ & $2(17)$ & $7(28)$ & $42(27)$ & \\
\hline Property & $2(8)$ & $2(17)$ & $5(20)$ & $29(19)$ & \\
\hline Sex & $0(0)$ & I (8) & 0 & $15(10)$ & \\
\hline Other & $3(12)$ & $2(17)$ & $5(20)$ & $14(9)$ & \\
\hline \multicolumn{6}{|c|}{ Number of court appearances 4} \\
\hline Median (range) & $1.0(0-38)$ & $5.0(0-16)$ & $3.0(0-2 I)$ & $3.0(0-4 I)$ & NS \\
\hline Mean/s.d. & $6.9 / 14.0 ; n=7$ & $5.4 / 5.3 ; n=10$ & $4.9 / 5.7 ; n=19$ & $4.4 / 5.5 ; n=127$ & NS \\
\hline \multicolumn{6}{|c|}{ Number of custodial sentences 4} \\
\hline Median (range) & $0(0-27)$ & $0.5(0-8)$ & $0(0-13)$ & $0(0-20)$ & NS \\
\hline Mean/s.d. & $4.6 / 10.1$ & $\mathrm{I} .5 / 2.5$ & I.7/3.3 & I.I/2.5 & 0.05 \\
\hline \multicolumn{6}{|c|}{ Type of detention order, $n(\%)$} \\
\hline Civil & II (48) & $2(18)$ & $3(12)$ & $25(16)$ & \\
\hline Unrestricted criminal & I (4) & I (9) & $4(16)$ & $21(14)$ & \\
\hline Restricted criminal & II (48) & $8(73)$ & $18(72)$ & $106(70)$ & 0.03 \\
\hline
\end{tabular}

MHA, Mental Health Act 1983.

I. Of those leaving special hospital in 1984, 8 are not included in this table because no follow-up information could be traced

2. Those with continuous residency in an institution until death or 12 year census.

3. Those who lived at some stage outside an institution.

4. Prior to special hospital admission. 
hospital residence occurred in 13 cases (mental illness to personality disorder in 5 of these) and probably accounted for this deviation from discharge findings, even though in statistical terms the disorder classes could be treated as the same at admission as at discharge (kappa $=0.9)$ (Table 2).

Neither seriousness of index offence nor seriousness of previous criminal career in terms of the measures shown in Table 2 were significantly associated with failure to return to the community. The type of MHA detention order was so associated, however, with civil detention accounting for proportionately more continuously institutionalised cases. (A civil order generally indicated a long career of violent, disruptive and other dangerous, noncriminalised behaviour in previous hospital placements.)

Logistic regression was used to examine whether or not community status was attained (regardless of death). No independently significant effects of age, MHA disorder classification on admission or gender were found.

\section{Facilities used by those who lived outside high-security hospital but did not return to community residence}

The total follow-up time available for those who left high-security hospital alive, but did not return to community residence, was 219 patient-years. The real follow-up time was shortened to 169 patient-years because of the 7 deaths in this group. Therefore the deaths foreshortened the available time by $23 \%$.

Table 3 shows that, for those people who did not live in the community again, most time was spent in ordinary general psychiatric hospitals. Time back in special hospital was second in duration for those who were still alive at the end of the study. Purpose-designed, specialist medium secure units had almost no part to play for the longer-term care of this group, and people were rarely returned to prison. (The 4 returning to prison were on sections $47 / 49$ $(n=3)$ or were charged with a new offence while still under sections 37/41 ( $n=1)$.)

For patients who did reach the community (whether or not they died there), two-thirds of the available time was spent in the community. About one-quarter to one-sixth of the time was spent in ordinary general psychiatric hospitals, and much less in readmission to special hospital. Very little time was spent in regional secure units or prison.

Table 3 shows that the distribution of type of institutional time differed between the groups. This is mainly accounted for by the short time spent back in special hospital by the groups of people who died (within or outside institutions) and the proportionately longer time by the continuously institutionalised group. The group still alive at the end of the study, but with community living experience, had collectively spent more time in prison - but this nevertheless occupied a small part of the time.

\section{Readmission to special hospital}

Readmissions to high-security hospital were over twice as likely in the continuously institutionalised group than in the group reaching the community at some point: 7/19 (37\%) v. 31/179 (17\%). Readmissions typically occurred soon after discharge (or departure). There was no significant difference in the median survival times to readmission between the three groups $(P=0.06)$ : continuously institutionalised group (1.79 years, $0.41-5.34$ years); 'alive-in-the-community' group (1.33 years, 0.02-8.47 years); 'dead-in-the-community' group (2.52 years, $1.85-3.53$ ).

One man in the continuously institutionalised group had had a further trial in lesser security, which had broken down, resulting in a second admission. Deteriorating psychosis ( 3 cases), violent behaviour ( 3 cases) and repeated absconding (1 case) accounted for the returns in the continuously institutionalised group. Assaultive behaviour of various kinds was the most usual reason for return to high security

Table 3 Facilities used over the 12 years (including trial leave episodes)

\begin{tabular}{|c|c|c|c|c|c|c|}
\hline \multirow[t]{2}{*}{ Nature of placement } & \multicolumn{3}{|c|}{ Died in institution $(n=7)$} & \multicolumn{3}{|c|}{ Alive but institutionalised $(n=12)$} \\
\hline & $n$ & Total years & $\%$ patient-years & $n$ & Total years & $\%$ patient-years \\
\hline Readmitted to special hospitals & 0 & 0 & 0 & 7 & 59.57 & 43 \\
\hline Medium-security hospital unit & 0 & 0 & 0 & 4 & 6.86 & 5 \\
\hline General psychiatric or other hospitals & 6 & 27.99 & 92 & 8 & 67.66 & 49 \\
\hline Prison & I & 2.46 & 8 & 3 & 4.08 & 3 \\
\hline Total & & 30.45 & & & 138.17 & \\
\hline
\end{tabular}

\begin{tabular}{|c|c|c|c|c|c|c|}
\hline & \multicolumn{3}{|c|}{ Died in community $(n=25)$} & \multicolumn{3}{|c|}{ Alive in community $(n=154)$} \\
\hline & $n$ & Total years & $\begin{array}{c}\text { Patient-years as \% } \\
\text { of institutional } \\
\text { time }\end{array}$ & $n$ & Total years & $\begin{array}{c}\text { Patient-years as \% } \\
\text { of institutional } \\
\text { time }\end{array}$ \\
\hline Readmitted to special hospitals & 3 & 5.38 & 12 & 28 & 148.56 & 26.5 \\
\hline Medium-security hospital unit & 0 & 0 & 0 & 28 & 37.24 & 6.6 \\
\hline General psychiatric or other hospitals & 14 & 37.49 & 83.4 & 86 & 290.91 & 51.9 \\
\hline Prison & 2 & 2.09 & 4.6 & 37 & 84.33 & 15 \\
\hline Total & & 44.96 & & & 561.04 & \\
\hline
\end{tabular}


in the community group, with a few readmissions for mental health reasons or absconding behaviour.

Of the 7 readmitted to high security from the continuously institutionalised group, 6 were still resident in special hospital at the end of the study. One man had a second readmission. The median readmission time was 9.15 years (4.4810.76 years). This meant that the median total length of time in special hospital for this group (to the end of 1995 only, inclusive of previous admissions) was 17.08 years $(9.68-31.72$ years), a figure very much higher than the median for men within the 1984 discharge cohort as a whole, which was 7.02 years (0.02-42.74 years).

\section{Reconvictions after high-security hospital discharge}

Overall, $69(36 \%)$ of the 192 people for whom criminal records were traced had reoffended, not necessarily seriously. Reoffending certainly did not occur in the tiny group (6) who left high-security hospital but died in another institution; however, 4 of the 18 (one not known) (22\%) who were surviving in institutions had reoffended. Nevertheless, this was a smaller proportion than that of the reoffending subgroup $(65,37 \%)$ among those who had at some point reached the community $\left(\chi_{3}^{2}=11.7\right.$, $P=0.008$ ).

These findings thus disproved the hypothesis that reoffending would be uniquely associated with community living. The 4 institutionalised men who were convicted of a criminal offence were living in non-secure hospitals at the time. Their offences, all serious and three committed outside the hospital, played a part in their continued failure to attain community living. Of these, 3 men ( 2 suffering from mental illness and 1 from psychopathic disorder) were readmitted to special hospital, and 1 received a prison sentence. It was unclear why a prison sentence had been handed down to this man, who was manifestly psychotic at the time of the offence and did not cope with his imprisonment; he lost remission for fighting. The 4 did not have extensive previous criminal records compared with their 14 chronically institutionalised peers. Before special hospital admission, 2 did not have an index offence; 1 of the other 2 had been admitted after a non-violent offence; and the fourth had an index offence of arson.

\section{DISCUSSION}

\section{An improvement in the residual institutionalised population?}

The principal hypothesis, that under a range of legal and service conditions established in or since 1984, all patients leaving high-security hospital would eventually return to community living, was disproved. If the special hospital deaths in 1984 were included, nearly one-fifth of this discharge cohort failed to return to the community and 3 further people never progressed beyond 24-hour-staffed hostels. Of the 12 people continuously institutionalised but alive at the census point, 4 had been readmitted to special hospital after further serious offences and seemed unlikely to return to the community. By definition the other 8 had been continuously institutionalised for 12 years since their high-security hospital discharge. This was so much longer than the median length of alternative institutional residence of 2.2 years (0.0611.03 years) for the community group that this residual group of institutionalised patients are therefore reasonably construed as people who 'do not return to the community'.

A more positive construction from the patients' perspective is that, of the highsecurity hospital patients discharged to other facilities, only $10 \%$ failed to reach community residence. This is a lower proportion than in Norris's (1984) study $(22 \%)$, suggesting a possibility that, in the intervening years, the increased scope for challenge to detention, increased range of service provision, or both, might have had some impact. The Norris follow-up time, however, was shorter (7 years).

\section{Implications for service planning}

For all practical purposes, once accepted for treatment in a high-security hospital, patients may expect one of three longerterm outcomes on discharge: return directly to the community (for a minority $-38 \%$ in 1984 ); return to the community after a period of residence in other hospitals; or indefinite residence in hospital, inclusive of return to special hospital in about one-third of these cases. This minority of special hospital returns ( $17 \%$ overall) is similar to that found in most other studies (Tong \& Mackay, 1959; Gathercole et al, 1968; Bailey \& MacCulloch, 1992). The exception is a study from one large secure unit in
England (Cope \& Ward, 1993), which reported the return of one-third of cases received there.

Medium secure hospital provision in the UK is currently designed for patients needing a period of residence of up to 2 years, although a few stay longer. Our study suggests that current medium secure provision has an insignificant long-term role for progressing high-security patients, and that they can be managed in general psychiatric units. Our data do not suggest that this balance of service use is inappropriate, or that there is a case to be made from data on departing highsecurity hospital patients for the creation of yet another new category of service. The concept of long-term medium-security hospital provision has become popular in the UK, but it is a concept with little refinement (Taylor et al, 1996). For people who can be managed without security, whether in the community or in open hospitals, there can be no case for longterm medium security. In our series, 4 men did offend while resident in an open hospital, but there was no evidence that they could not have been managed within the present service organisations with more flexible use of current medium security, or even low security. The very small numbers of patients in the continuously institutionalised group who returned to high security did not appear to constitute a demand for a whole new tier of service, even if repeated for each discharge cohort. Then, as those not reaching the community were more likely to have a psychotic illness than any other condition, improvements in anti-psychotic medication might be expected to reduce the size of the residual institutionalised group further.

\section{Gender}

The finding that it was mainly men with mental illness who were continuously institutionalised is comparable with findings among patients in general psychiatry services (Lelliott et al, 1994). This too was a national study, drawing data from psychiatrists in 59 services in the UK. The patients were between 18 and 64 years of age on admission, and had been hospitalised for between 6 months and 3 years. New long-stay subjects were younger (1834 years), predominantly single men with schizophrenia; $43 \%$ of these had a history of serious violence, dangerous behaviour 
or admission to a special hospital, and over one-third were formally detained.

\section{Limitations and directions for future research}

Collection of data for this type of study is difficult and very time-consuming, and there is a loss of traces over the years. It does, however, offer some gain in knowledge for service planning.

Some subgroups of interest examined here are very small. Furthermore, simple concepts of community or institutional residence are not adequate for understanding the whole picture in some cases. Some people living in hospitals appeared to have been spending much of their days outside those hospitals in the wider community, whereas patients in some of the hostel placements (designated community placements) appeared to be more supervised and observed than those in some hospitals. From the records available, however, it would seem that the recording of data was not significantly consistent to quantify these issues further. A prospective study with greater clarity on the nature and intensity of observation, supervision, 'asylum' and support is indicated.

\section{REFERENCES}

Bailey, J. \& MacCulloch, M. (1992) Characteristics of II2 cases discharged directly to the community from a new special hospital and some comparisons of performance. Journal of Forensic Psychiatry, 3, 91-112.

Buchanan, A. (1998) Criminal conviction after discharge from special (high security) hospital. Incidence in the first 10 years. British Journal of Psychiatry, I72, $472-476$.

Butwell, M., Jamieson, E., Leese, M., et al (2000) Trends in special (high-security) hospitals. 2: Residency and discharge episodes, 1986-1995. British Journal of Psychiatry, 176, 260-265.

Cope, R. \& Ward, M. (1993) What happens to special hospital patients admitted to medium security? Journal of Forensic Psychiatry, 4, 13-24.

Gathercole, C. E., Craft, M. J., McDougall, J. M., et al (1968) A review of 100 discharges from a special hospital. British Journal of Criminology, 8, 419-424.

\section{CLINICAL IMPLICATIONS}

- It is the combination of older age and mental illness rather than offending or personality disorder which appears to limit return to the community for patients who have been deemed a risk to the public.

- Men are especially vulnerable to indefinite institutionalisation.

- For those who continued to use institutions, the institution was almost invariably a hospital and they either stayed in low security or returned to high security. There is no clear case for a new tier of long-term medium security.

\section{LIMITATIONS}

Data collection for this type of study is very time consuming and there is attrition in capacity to trace cases over time.

- The census point of 12 years was imposed on the follow-up, and it may be that some of the 12 men remaining in institutions later reached the community.

- Details of individual needs and the extent to which these were or were not met were beyond the scope of this study.

LIZ JAMIESON, BSc, Broadmoor Hospital, Crowthorne, Berkshire; PAMELA J. TAYLOR, FRCPsych, Institute of Psychiatry, London, UK

Correspondence: Liz Jamieson, Lecturer, Broadmoor Hospital, Crowthorne, Berkshire RG45 7EG, UK

(First received 9 July 200I, final revision 23 May 2002, accepted 26 June 2002)

Home Office \& Department of Health and Social Security (1975) Report of the Committee on Mentally Abnormal Offenders (The Butler Report) (Cm 6244). London: $\mathrm{HMSO}$

Jamieson, E., Butwell, M., Taylor, P. J., et al (2000) Trends in special (high-security) hospitals. I: Referrals and admissions 1986-1995. British Journal of Psychiatry, 176, 253-259.

Lelliott, P., Wing, J. \& Clifford, P. (1994) A nationa audit of new long-stay psychiatric patients. I: Method and description of the cohort. British Journal of Psychiatry, 165, 160-169.

Norris, M. (1984) Integration of Special Hospital Patients into the Community. Aldershot: Gower.
Snowden, P. R. (1985) A survey of the Regional Secure Unit programme. British Journal of Psychiatry, 147 499-507.

StataCorp (1997) Stata Statistical Software. Release 6.0. College Station, TX: Stata Corporation.

Taylor, P. J., Maden, A. \& Jones, D. (1996) Long term medium security hospital units: a service gap of the 1990s? Criminal Behaviour \& Mental Health, 6, 213-229.

_ , Leese, M., William, D., et al (1998) Mental disorder and violence. A special (high-security) hospital study. British Journal of Psychiatry, 172, 218-226.

Tong, J. E. \& Mackay, G.W. (1959) A statistical follow-up of mental defectives of dangerous or violent propensities. British Journal of Delinquency, 9, 276-284. 